\title{
Combined interstitial laser cauterisation of placental anastomosis and intrauterine intracardiac transfusion following monochorionic co-twin demise: a case report
}

PW Hui *, MD, FRCOG, Mimi TY Seto, MB, BS, FHKAM (Obstetrics and Gynaecology), KW Cheung, MB, BS, FHKAM (Obstetrics and Gynaecology)

Department of Obstetrics and Gynaecology, Queen Mary Hospital, Hong Kong

*Corresponding author: apwhui@hku.hk

Hong Kong Med J 2021;27:293-6

https://doi.org/10.12809/hkmj208806

\section{Case report}

Single fetal demise in monochorionic pregnancy is associated with significant morbidity and mortality of the co-twin. We report the case of a 36-year-old nulliparous woman with unexpected single fetal demise in a monochorionic twin pregnancy diagnosed at 15 weeks and 4 days. She had been well since her last normal scan at 13 weeks and 3 days and no discordance in crown rump length or nuchal translucency thickness had been evident. Noninvasive prenatal testing for common aneuploidy screening was negative.

Ultrasound showed demise of one twin and scalp oedema and ascites in the surviving co-twin. Cardiothoracic ratio was elevated to 0.59 and tricuspid regurgitation was seen. Peak systolic velocity (PSV) of the middle cerebral artery (MCA) was increased to 2.18 multiples of median (MoM; $45.1 \mathrm{~cm} / \mathrm{s}$ ) and diastolic flow in the umbilical artery was absent. Cord insertion was velamentous and an arterial anastomosis was identified along the placental surface from the surviving twin to the placental cord insertion of the miscarried twin (Fig $1 \mathrm{a}$ and $\mathrm{b}$ ).

The couple were counselled extensively on management options that included termination of pregnancy, conservative management, or active intrauterine interventions. Within 24 hours, the patient opted to undergo rescue transfusion for fetal anaemia and interstitial laser cauterisation of the placental anastomosis. Interstitial laser cauterisation was performed using an 18G spinal needle inserted transplacentally under ultrasound guidance to the anastomotic artery close to the cord insertion of the miscarried twin. A 780- $\mu \mathrm{m}$ laser fibre was advanced to $3 \mathrm{~mm}$ beyond the needle tip. Cauterisation was started with a $20-\mathrm{W}$ diode laser and stepped up to $40 \mathrm{~W}$ for $100 \mathrm{~s}$. Fetal heart pulsation was checked intermittently and was normal throughout. Cessation of blood flow was confirmed by colour Doppler ultrasonography (Fig 1c).

Intracardiac transfusion was performed the next day via a $22 \mathrm{G}$ needle directed to the fetal right ventricle (Fig 1d). Before the transfusion, haemoglobin was $4.2 \mathrm{~g} / \mathrm{dL}$ and haematocrit was $10.5 \%$. Transfusion of $4.5-\mathrm{mL} \mathrm{O}$ positive blood with haematocrit of $80 \%$ and irradiated for cytomegalovirus was uneventful. No bradycardia or pericardial effusion occurred. After the transfusion, haemoglobin was $12.6 \mathrm{~g} / \mathrm{dL}$ and haematocrit was $37.2 \%$. The MCA PSV was $48.7 \mathrm{~cm} / \mathrm{s}(2.35 \mathrm{MoM})$ before the laser cauterisation and $50 \mathrm{~cm} / \mathrm{s}$ (2.39 MoM) before the transfusion. It was reduced to $0.9 \mathrm{MoM}$ after the transfusion. Other workup for fetal hydrops was negative.

The patient was monitored by weekly ultrasound for level of MCA PSV MoM and resolution of hydrops. Ascites disappeared 5 days after transfusion and MCA PSV was 1.3 MoM. Subcutaneous oedema subsided 1 week later and cardiothoracic ratio improved to 0.56 . Fetal magnetic resonance imaging and detailed echocardiography at 22 weeks of gestation showed no abnormality. A loop of prominent bowel was noticed after 23 weeks of gestation. This was progressively dilated to $1.72 \mathrm{~cm}$ at 33 weeks of gestation. Peristalsis was present and bowel atresia was suspected. The patient went into preterm labour at 34 weeks and 2 days, delivering a baby boy weighing $1960 \mathrm{~g}$. An area of cauterisation on the placental surface was evident next to the cord insertion of the miscarried twin (Fig 2).

Neonatal laparotomy on day 1 showed type 4 intestinal atresia. Resection of multiple atretic segments of the small bowel and primary anastomosis were performed. A second laparotomy was required on day 34 for resection of rectal atresia. Bowel function returned to normal afterwards. The baby had grade 1 intraventricular haemorrhage that resolved spontaneously and at age 5 months he had reached the appropriate developmental milestones.

\section{Discussion}

Single twin demise occurs in monochorionic pregnancies with twin-twin transfusion syndrome 


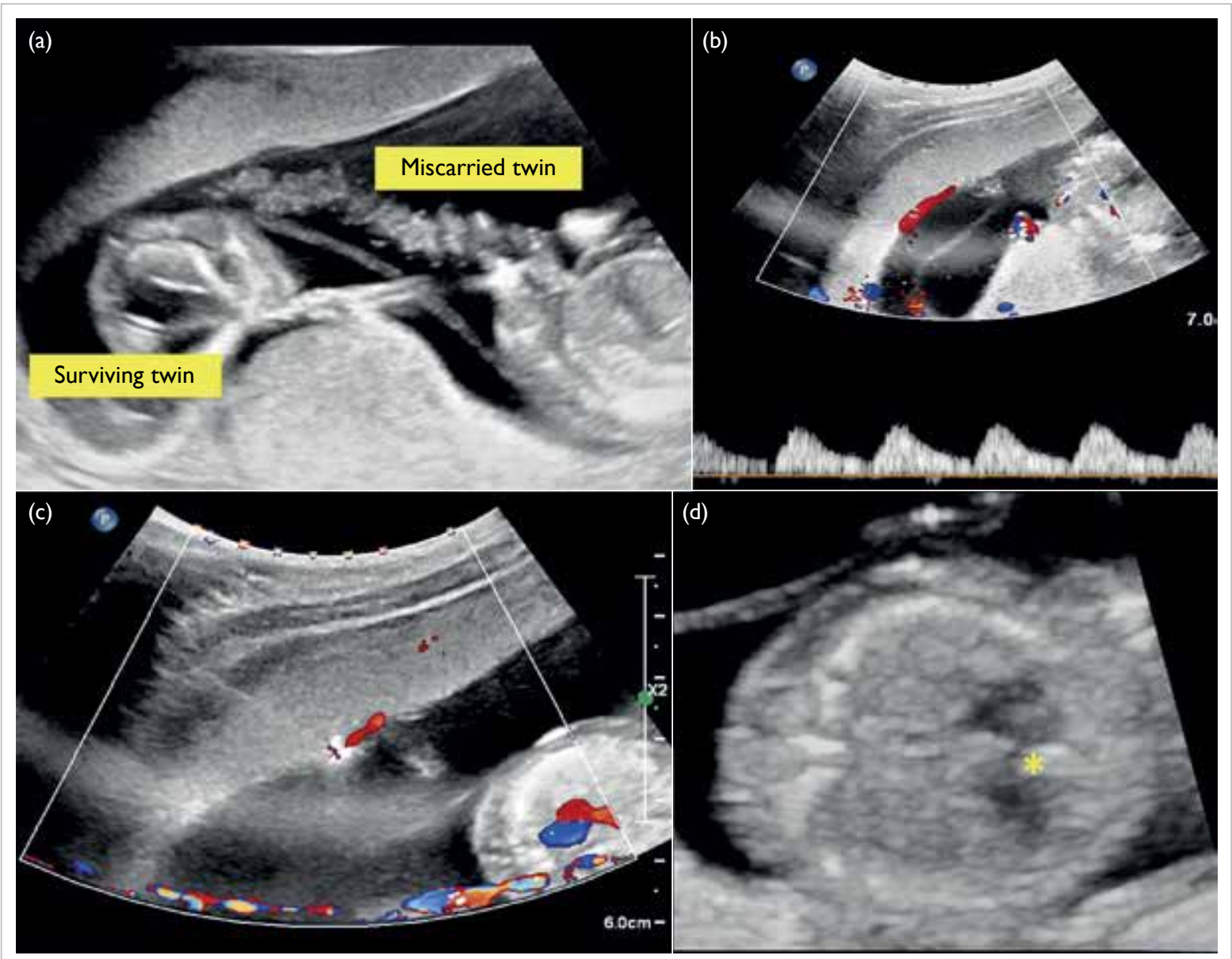

FIG I. Ultrasound images showing the presence of arterial placental anastomosis from the surviving twin to placental cord insertion of the miscarried twin, laser cauterisation and intracardiac transfusion. (a) Placental cord insertion of miscarried twin; (b) arterial vessel running from surviving twin to placental cord insertion of miscarried twin; (c) laser cauterisation of arterial anastomotic vessel; and (d) intracardiac transfusion with needle directed to right ventricle

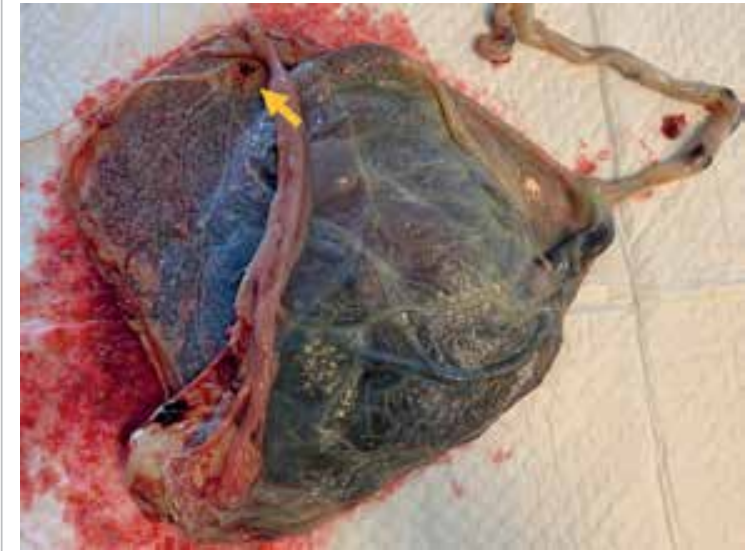

FIG 2. Photograph of placenta showing an area of cauterisation (arrow) next to cord insertion of miscarried twin

(TTTS) and selective intrauterine growth restriction but may also occur unexpectedly in pregnancies with no obvious complications. ${ }^{1}$ Feto-fetal haemorrhage through the placenta anastomosis leads to acute hypovolaemia and results in fetal anaemia, cerebral damage or death of the co-twin. ${ }^{1,2}$ Bowel and renal complications have also been reported. ${ }^{3,4}$ The present case illustrates successful intervention with a combination of interstitial laser to a placental anastomosis and intracardiac transfusion for a fetus at 15 weeks of gestation following monochorionic co-twin demise. The risk of co-twin demise and neonatal death is increased significantly in cases of single intrauterine fetal death in monochorionic pregnancies before 28 weeks. ${ }^{5}$ The surviving fetus is also at risk of other morbidity secondary to fetofetal haemorrhage and hypoperfusion. Neurological damage affects almost $20 \%$ of co-twin survivors especially in fetuses demonstrating signs of fetal anaemia., ${ }^{1,6}$ This can be assessed by measuring MCA PSV. ${ }^{7}$ A value $>1.55 \mathrm{MoM}$ is suggested to be associated with a fivefold increase in the relative risk of cerebral injury. ${ }^{1}$

Rescue intrauterine transfusion has been proposed for an anaemic monochorionic survivor. ${ }^{8}$ The optimal timing of transfusion and impact on 
overall long-term outcome remain debatable. Fetofetal haemorrhage may continue after intrauterine transfusion. It is, therefore, rational to conduct a sonographic search for placental anastomosis and perform cauterisation to cease further feto-fetal haemorrhage prior to intrauterine transfusion. In this case, an arterial vessel running towards the placental cord insertion of the miscarried twin was identified. With the presence of this anastomosis, rescue transfusion for the surviving fetus was unlikely to be effective as fetal anaemia could persist. Interstitial laser has been reported in cauterisation of feeding vessels in chorioangioma, fetal hyperechogenic lung lesion, and sacrococcygeal teratoma. ${ }^{9}$ Adopting the principle of intrauterine laser ablation in TTTS, a laser fibre was inserted via a $18 \mathrm{G}$ spinal needle transplacentally to cauterise this vessel under ultrasound guidance. A fetoscopic approach was not considered in view of the early gestation with an anterior placenta and absence of polyhydramnios. The energy requirement was guided by generation of an echogenic area around the fibre tip encompassing the vessel and cessation of blood flow on Doppler ultrasound.

Intracardiac fetal transfusion was performed because of the early gestation and difficult intravascular assess. This technique was first introduced in the late 1980s. ${ }^{10}$ Experience was gathered mainly from transfusing fetuses that were anaemic due to rhesus isoimmunisation or parvovirus infection reported as early as 16 weeks of gestation..$^{11-13}$ Transient fetal bradycardia, haemopericardium, pericardial tamponade, and asystole are known complications. ${ }^{10-12}$

To the best of our knowledge, this is the first report of successful rescue of an anaemic co-twin survivor in a monochorionic pregnancy by combining laser to placental anastomosis and intracardiac transfusion as early as 15 weeks of gestation. Time was required for blood product preparation and also allowed the fetus to establish a new circulatory equilibrium following laser therapy when the ongoing transfusion was completed. It is of interest to note that fetal ascites may be an early presentation of bowel complications secondary to in-utero anaemia. ${ }^{3}$ Mesenteric ischaemia in monochorionic twins has been postulated to be related to haemodynamic alteration in case of co-twin demise, hypoperfusion, and/or hyperviscosity in TTTS or thromboembolic phenomenon after laser ablation. Ascites might also be an indication of circulatory insufficiency even in the absence of severe fetal anaemia. Evidence of transient cardiac failure has been reported after single fetal death in monochorionic twins and immediate intrauterine transfusion has been advocated to restore the circulatory volume. ${ }^{2}$

This case did not have features of TTTS although ascites, cardiomegaly, and tricuspid regurgitation were noted after co-twin demise and before intrauterine intervention. Although fetal anaemia was ascertained and hypovolaemic shock was likely present, it is not always possible to determine whether the ascites is secondary to circulatory insufficiency or bowel complications. As well as treating fetal anaemia, intrauterine transfusion may also correct circulatory insufficiency and restore cardiac function. The prompt intervention in this fetus led to complete resolution of the ultrasound abnormalities and possibly improved the neurological outcomes. The fetus required further monitoring for bowel complications that might only become obvious at a later stage of gestation.

In managing co-twin demise in a monochorionic pregnancy, assessment of the survivor requires detailed ultrasound examination to search for placental anastomosis, as well as an immediate assessment for signs of fetal anaemia and bowel or haemodynamic complications. Early laser cauterisation of placental anastomosis to control feto-fetal haemorrhage is an option combined with rescue intrauterine transfusion to prevent anaemia and circulatory insufficiency in the surviving twin.

\section{Author contributions}

All authors contributed to the concept of the study, acquisition of the data, and analysis of the data, and critical revision of the manuscript for important intellectual content. PW Hui drafted the manuscript. All authors had full access to the data, contributed to the study, approved the final version for publication, and take responsibility for its accuracy and integrity.

\section{Conflicts of interest}

All authors have disclosed no conflicts of interest.

\section{Funding/support}

This study received no specific grant from any funding agency in the public, commercial, or not-for-profit sectors.

\section{Ethics approval}

The patient was treated in accordance with the Declaration of Helsinki and provided informed consent for all procedures.

\section{References}

1. Lanna MM, Consonni D, Faiola $S$, et al. Incidence of cerebral injury in monochorionic twin survivors after spontaneous single demise: long-term outcome of a large cohort. Fetal Diagn Ther 2020;47:66-73.

2. Iwagaki S, Takahashi Y, Chiaki R, Asai K, Matsui M, Katsura D. Case of resuscitation from cardiac failure by intrauterine transfusion after single fetal death in monochorionic twin pregnancy. J Obstet Gynaecol Res 2019;45:2105-10.

3. Tan LN, Cheung KW, Philip I, Ong S, Kilby MD. Isolated ascites in a monochorionic twin after fetoscopic laser ablation is not necessarily secondary to recurrence or 
anaemia: bowel complications in twin-to-twin transfusion syndrome after fetoscopic laser ablation. Fetal Diagn Ther 2019;45:285-94.

4. Genova L, Sueters M, van Steenis A, Oepkes D, Steggerda SJ, Lopriore E. Renal failure after single fetal demise in monochorionic twins: incidence and description of a case. Fetal Diagn Ther 2014;35:302-5.

5. Mackie FL, Rigby A, Morris RK, Kilby MD. Prognosis of the co-twin following spontaneous single intrauterine fetal death in twin pregnancies: a systematic review and metaanalysis. BJOG 2019;126:569-78.

6. Mackie FL, Morris RK, Kilby MD. Fetal brain injury in survivors of twin pregnancies complicated by demise of one twin: a review. Twin Res Hum Genet 2016;19:262-7.

7. Senat MV, Loizeau S, Couderc S, Bernard JP, Ville Y. The value of middle cerebral artery peak systolic velocity in the diagnosis of fetal anemia after intrauterine death of one monochorionic twin. Am J Obstet Gynecol 2003;189:1320 4.

8. Quarello E, Stirnemann J, Nassar M, et al. Outcome of anaemic monochorionic single survivors following early intrauterine rescue transfusion in cases of feto-fetal transfusion syndrome. BJOG 2008;115:595-601.

9. Mathis J, Raio L, Baud D. Fetal laser therapy: applications in the management of fetal pathologies. Prenat Diagn 2015;35:623-36

10. Westgren M, Selbing A, Stangenberg M. Fetal intracardiac transfusions in patients with severe rhesus isoimmunisation. Br Med J (Clin Res Ed) 1988;296:885-6.

11. von Kaisenberg CS, Grebe S, Schleider S, Kuhling-von Kaisenberg H, Venhoff L, Meinhold-Heerlein I. Successful intrauterine intracardiac transfusion in monochorionic twins affected by parvovirus B19. Fetal Diagn Ther 2007;22:420-4.

12. Mackie FL, Pretlove SJ, Martin WL, Donovan V, Kilby MD. Fetal intracardiac transfusions in hydropic fetuses with severe anemia. Fetal Diagn Ther 2015;38:61-4.

13. Yinon Y, Visser J, Kelly EN, et al. Early intrauterine transfusion in severe red blood cell alloimmunization. Ultrasound Obstet Gynecol 2010;36:601-6. 